\title{
Study of Optimal Operation for Huai'an Parallel Pumping Stations with Adjustable-Blade Units Based on Two Stages Decomposition-Dynamic Programming Aggregation Method
}

\author{
Yi Gong ${ }^{1}$, Jilin Cheng ${ }^{1}$, Rentian Zhang ${ }^{1,2}$, and Lihua Zhang ${ }^{1}$ \\ ${ }^{1}$ College of Hydraulic Science and Engineering, Yangzhou University, Yangzhou, China \\ ${ }^{2}$ Jiangsu Surveying and Design Institute of Water Resources Co., Ltd., Yangzhou, China \\ gongyi_8@163.com, jlcheng@yzu.edu.cn, r_zhang@yzu.edu.cn, \\ lhzhang@yzu.edu.cn
}

\begin{abstract}
Two-stage decomposition-dynamic programming aggregation method has been first proposed and introduced to solve the mathematical model of daily optimal operation for parallel pumping stations with adjustable-blade units. Taking minimal daily electricity cost of single pump station as objective function, the water quantity pumped by each station as coordinated variable, by means of the type of the pump units this model is decomposed into several first-stage sub-model of daily optimal operation with adjustable-blade for single pump station. Then taking minimal daily electricity cost of single pump unit as objective function, the water quantity pumped by each unit as coordinated variable, the first-stage sub-model is decomposed into several second-stage sub-model of daily optimal operation with adjustable-blade for single pump unit which takes the blade angle as decision variable, the discrete values of water quantity pumped by each unit as state variable, and is solved by means of dynamic programming method. The constructed aggregation model takes daily water quantity pumped by each pump unit as decision variable, the discrete values of water quantity pumped by parallel station group as state variable, and is also solved by dynamic programming method. The aggregation process replaces the traditional method of constructing equations. This method has first solved the optimal operation issues for multi-units of parallel stations with various operation modes, time period division and daily average head of each station, and also provided theoretical support for the study on optimal operation of multi-stage pumping stations. Taking Huai'an No.1, No.2, and No.4 parallel pumping stations as a study case, a series of optimization results have been obtained.
\end{abstract}

Keywords: parallel pumping stations, decomposition, aggregation, dynamic programming, optimization, adjustable-blade.

\section{Introduction}

The parallel pumping stations have a huge energy consumption in operation because of containing a large number of pump units, which makes it necessary to develop the study of optimal operation method for multi-units of parallel pumping stations. At 
present, the major study methods of optimal operation for parallel pumping stations contain decomposition-coordination method and decomposition-aggregation method. The former would bring tedious combinations while more pump units exist. And the latter always establishes regression equation while in aggregation process, which would affect the solution precision of optimization model. Therefor, two-stage decomposition-dynamic programming aggregation method has been proposed and applied to the study. Taking No.1, No.2 and No.4 Huai,an Pumping Station as a study case, we divide one day into several time periods according to the inlfluence of peak-valley electricityity price and the demands of not high frequency start-up/shut down operation, by which to search the optimization benefit carried out by optimal operation of multi-units with adjustable-blade in parallel pumping stations operation.

\section{Optimal Daily Operation Model and Its Solving Method for Multi-units with Adjustable-Blade in Parallel Pumping Stations}

In order to be convenient for discussion, a series of definitions have been made which are as follows:

(1) Operation mode

Operation with fixed blade angle and constant speed: The pump units are operating with rating speed and the blade angle at the designing degree.

Optimal operation with adjustable-blade: The pump units are operating with constant speed and adjusting the blade angle in each time period according to operation conditions in order to obtain the minimal water pumping cost.

(2) Full load, $80 \%$ load, $60 \%$ load

The operation of pump units lasting 24 durative hours is called full load operation. $80 \%$ load operation and $60 \%$ load operation respectively represents the pump units are operating with $80 \%$ and $60 \%$ of water quantity pumped by the units while they are operating with full load at fixed blade angle and constant speed.

(3) Beginning time and the combination between length of time period and peak-valley electricityity price

Considering peak-valley electricityity price and the demand of avoiding high frequency of start-up/shut down operation, we choose beginning time at $17: 00$ and divide one day into 9 periods. The time length and the electricityity price in each of period is shown in Table 1.

\subsection{Optimal Model of Daily Operation for Multi-units with Adjustable-Blade in Parallel Pumping Stations}

Taking minimal daily electricity cost of entire parallel pumping group as objective function, the time period as stage variable, the blade angle of each pump unit in each time period as decision variable, the water quantity pumped in definite time period and power of electromotor equipped in station as the constraint conditions, the optimal mathematical model of daily operation for multi-units with adjustable-blade in parallel pumping stations has been construced as follows : 
Table 1. Time period division and peak-valley electricity price of each time period

\begin{tabular}{cccccccc}
\hline $\begin{array}{c}\text { Serial } \\
\text { number }\end{array}$ & Time division & $\begin{array}{c}\text { Length } \\
\text { of time } \\
\text { period/h }\end{array}$ & $\begin{array}{c}\text { Electricityity } \\
\text { price/yuan. } \\
\mathrm{kW}^{-1} \cdot \mathrm{h}^{-1}\end{array}$ & $\begin{array}{c}\text { Serial } \\
\text { number }\end{array}$ & Time division & $\begin{array}{c}\text { Length } \\
\text { of time } \\
\text { period/h }\end{array}$ & $\begin{array}{c}\text { Electricityity } \\
\text { price/yuan· } \\
\mathrm{kW}^{-1} \cdot \mathrm{h}^{-1}\end{array}$ \\
\hline I & $17: 00 \sim 19: 00$ & 2 & 0.978 & VI & $07: 00 \sim 09: 00$ & 2 & 0.978 \\
II & $19: 00 \sim 21: 00$ & 2 & 0.978 & VII & $09: 00 \sim 11: 00$ & 2 & 0.978 \\
III & $21: 00 \sim 23: 00$ & 2 & 0.587 & VIII & $11: 00 \sim 14: 00$ & 3 & 0.587 \\
IV & $23: 00 \sim 03: 00$ & 4 & 0.276 & IX & $14: 00 \sim 17: 00$ & 3 & 0.587 \\
V & $03: 00 \sim 07: 00$ & 4 & 0.276 & & & & \\
\hline
\end{tabular}

Objective function: $\quad G=\min \sum_{k=1}^{B Z} G_{k}=\min \left(\sum_{k=1}^{B Z} \sum_{j=1}^{J Z} \sum_{i=1}^{S N} \frac{\rho g Q_{k j i}\left(\theta_{k j i}\right) H_{k j i}}{\eta_{z, k j i} \eta_{m o t, k j} \eta_{i n t, k j}} \Delta T_{k i} P_{k i}\right)$

Water quantity constraint: $\quad \sum_{k=1}^{B Z} \sum_{j=1}^{J Z} \sum_{i=1}^{S N} Q_{k j i}\left(\theta_{k j i}\right) \Delta T_{i} \geq W_{e}$

Power constraint:

$$
N_{k j i}\left(\theta_{k j i}\right) \leq N_{k j 0}
$$

Where $G$ is the minimal daily electricity cost of the entire parallel pumping group. $G_{k}$ is the daily electricity cost of the $k$-th pumping station. BZ is quantity of pumping stations in the parallel pumping group. JZ is quantity of pump units in each single station. $S N$ is the quantity of time periods divided in one day. $\rho$ is water density and $\mathrm{g}$ is acceleration of gravity. $H_{k j i}$ and $Q_{k j i}\left(\theta_{k j i}\right)$ which is corresponding to the blade angle $\theta_{k j i}$ respectively represent the average daily head and flow of the $j$-th pump unit in the $k$-th pumping station and in the $i$-th time period. $\triangle T_{k i}$ and $P_{k i}$ respectively represent the time length and the peak-valley electricity price of the $i$-th time period in the $k$-th pumping station.

$\eta_{z, k j i}\left(\theta_{k j j}\right), \eta_{m o t k j,}, \eta_{i n t, k j}$ respectively represent the efficiency of equipment, electromotor and transmission of the $j$-th pump unit in the $k$-th pumping station. Among them $\eta_{z, k j i}$ is relative to the flow and average head of the $i$-th period. $\eta_{m o t, k j}$ could be regarded as constant when the load is over $60 \%$, while in large electromotor the $\eta_{m o t, k j}$ could be regarded as $94 \%$. Also we considered 1 as the $\eta_{i n t, k j}$ value in direct joint unit. $W_{e}$ is the objective water quantity pumped by the whole parallel pumping group in one day. And $N_{k j i}\left(\theta_{k j i}\right)$ is the actual electromotor power of the $j$-th pump unit in the $k$-th pumping station and in the $i$-th time period while the pump unit is operating under the blade angle $\theta_{k i}$, which should be less than the rating power of $N_{k j 0}$.

\subsection{Two-Stage Decomposition-Dynamic Programming Aggregation Method}

\subsubsection{Large-Scale Two-Stage Decomposition}

2.2.1.1 First-Stage Decomposition. Taking water quantity pumped by each pumping station as the coordinated variable, we decompose eq. (1) (3) into BZ first-stage subsystems according to the type of pump unit with the assumption that the units have the 
same type in the same pumping station. Then the optimal mathematical model of daily operation for multi-units with adjustable-blade for single pumping station is obtained which is shown from eq. (4) (6). This model takes minimal daily electricity cost of single pumping station as objective function, the blade angle of each pump unit in each time period as decision variable, the water quantity pumped in definite time period and power of electromotor equipped in station as the constraint conditions.

Objective function: $\quad F=\min \sum_{j=1}^{J Z} F_{j}=\min \left(\sum_{j=1}^{J Z} \sum_{i=1}^{S N} \frac{\rho g Q_{i, j}\left(\theta_{j i}\right) H_{j i}}{\eta_{z i, j}\left(\theta_{j i}\right) \eta_{m o t, j} \eta_{\text {int }, j}} \Delta T_{i} P_{i}\right)$

Water quantity constraint: $\quad \sum_{j=1}^{J Z} \sum_{i=1}^{S N} Q_{j i}\left(\theta_{i}\right) \Delta T_{i} \geq W_{k}$

Power constraint: $\quad N_{k j i}\left(\theta_{i}\right) \leq N_{k j 0}(i=1,2, \ldots, S N ; j=1,2, \ldots, J Z)$

Where $F$ is the minimal daily electricity cost of single pumping station. $F_{j}$ is daily electricity cost of the $j$-th pump unit. $W_{k}$ is the objective water quantity pumped by single pumping station in one day Meanings of other variables could be obtained by analogy according to eq. (1) (3).

2.2.1.2 Second-Stage Decomposition. Taking water quantity pumped by each pump unit as the coordinated variable, eq. (4) (6) is decomposed into JZ second-stage subsystems according to the quantity of pump unit in one single station. Then the optimal mathematical model of daily operation for single pump unit with adjustable-blade is obtained which is shown from eq. (7) (9). This model takes minimal daily electricity cost of single pump unit as objective function, the blade angle of each pump unit in each time period as decision variable, the water quantity pumped in definite time period and power of electromotor equipped in station as the constraint conditions. The blade angle is chosen at integer degree in order to be convenient for practical operation.

Objective function: $\quad M=\min F_{j}=\sum_{i=1}^{S N} \frac{\rho g Q_{i, j}\left(\theta_{i}\right) H_{i}}{\eta_{\mathrm{zi}, j} \eta_{\mathrm{mot}, j} \eta_{\mathrm{int}, j}} \Delta T_{i} P_{i} \quad(j=1,2, \ldots J Z)$

Water quantity constraint: $\quad \sum_{i=1}^{S N} Q_{j i}\left(\theta_{i}\right) \Delta T_{i} \geq W_{j} \quad(i=1,2, \ldots, S N ; j=1,2, \ldots J Z)$

Power constraint:

$$
N_{j i}\left(\theta_{i}\right) \leq N_{j 0}(i=1,2, \ldots, S N ; j=1,2, \ldots J Z)
$$

Where $M$ is the minimal daily electricity cost of single pump unit. $F_{j}$ is daily electricity cost of the $j$-th pump unit. $W_{j}$ is the objective water quantity pumped by single pump unit in one day. Meanings of other variables could be obtained by analogy according to eq. (1) (3).

\subsubsection{Optimization of Second-Stage Subsystem}

Eq. (7) (9) are typical one-dimension dynamic programming model whose stage variable is $i(i=1,2, \ldots, S N)$ and decision variable is the blade angle $\theta_{i}$. Also we could know from eq. (8) that the water quantity pumped in each time period is the state 
variable $\lambda$. Making use of dynamic programming method to solve this model, a series of $F_{j}$ values corresponding to objective water quantity $W_{j}$ could be obtained. The solving details of this model have been shown as follows:

Stage 1:

$$
M_{1}\left(\lambda_{1}\right)=\min \frac{\rho g Q_{1}\left(\theta_{1}\right) H_{1}}{\eta_{\mathrm{z} 1} \eta_{\mathrm{mot}} \eta_{\mathrm{int}}} \Delta T_{1} P_{1}
$$

The stage variable $\lambda_{1}$ is discrete within its feasible region: $\lambda_{1}=0, W_{1}, W_{2}, \cdots, W_{j}$. The decision variable $\theta_{1}$ is discrete within its feasible region for example: $-4^{\circ},-3^{\circ},-2^{\circ},-1^{\circ}$, $0^{\circ},+1^{\circ},+2^{\circ},+3^{\circ},+4^{\circ}$. Also the condition $Q_{1}\left(\theta_{1}\right) \Delta T_{1} \geq \lambda_{1}$ should be satisfied. According to the performance curve of pump device, the flow and equipment efficiency $\eta_{\mathrm{z} 1}$ corresponding to each $\theta_{1}$ and $H_{l}$ could be obtained.

Stage $i$ :

$$
g_{i}\left(\lambda_{i}\right)=\min \left[\frac{\rho g Q_{i}\left(\theta_{i}\right) H_{i}}{\eta_{z i} \eta_{\mathrm{mot}} \eta_{\mathrm{int}}} \Delta T_{i} \cdot P_{i}+g_{i-1}\left(\lambda_{i-1}\right)\right]
$$

$\lambda_{i}$ and $\theta_{i}$ are discrete in the same way as above. Also $Q_{i}\left(\theta_{i}\right) \Delta T_{i} \geq \lambda_{i}$ should be satisfied. According to eq. (8), the state transition equation is as follows:

$$
\lambda_{i-1}=\lambda_{i}-Q_{i}\left(\theta_{i}\right) \Delta T_{i}(i=2,3, \ldots, S N-1)
$$

Stage $S N$ :

$$
g_{S N}\left(\lambda_{S N}\right)=\min \left[\frac{\rho g Q_{S N}\left(\theta_{S N}\right) H_{S N}}{\eta_{z S N} \eta_{m o t} \eta_{\mathrm{int}}} \Delta T_{S N} \cdot P_{S N}+g_{S N-1}\left(\lambda_{S N-1}\right)\right]
$$

Where $\lambda_{S N}=W_{j}, W_{j+1} ; \theta_{S N=}-4^{\circ},-3^{\circ},-2^{\circ},-1^{\circ}, 0^{\circ},+1^{\circ},+2^{\circ},+3^{\circ},+4^{\circ}$

The state transition equation: $\lambda_{S N-1}=\lambda_{S N}-Q_{S N}\left(\theta_{S N}\right) \Delta T_{S N}\left(\lambda_{S N} \geq W_{j}\right)$

With the assumption that $B Z$ pump stations are contained in one parallel pumping group and the pump units contained in each pumping station have the same type with no performance difference while the pump units in different stations have different types, each pumping station has one kind of performance curve of pump unit. Each pump unit has a blade angle corresponding to the maximal flow within the power constraint under the average head of each time period. After taking a definite water quantity step to disperse the total water quantity $W_{j, \max }$ which corresponds to the maximal blade angle of all time periods, the optimal mathematical model of daily operation for single pump unit with adjustable-blade could be applied to calculate the minimal daily cost of single pump unit $F_{j, m}(m=1,2, \ldots$, max $)$ which respectively corresponds to each water quantity $W_{j, m}$.

With the fact that the pump units in one station have the same type with no performance difference, each pumping station only needs one optimal solution. Therefor, $B Z$ groups of optimal solution should be done in one parallel pumping group, after which $W_{k j m} \sim F_{k j m}\left(W_{k j m}\right)$ relationship could be obtained. 


\subsubsection{Dynamic Programming Aggregation of Large-Scale System}

After a series of $W_{k j m} \sim F_{k j m}\left(W_{k j m}\right)$ relationships are obtained by means of the second-stage submodel solutions $(k=1,2, \ldots, B Z ; j=1,2, \ldots, J Z ; m=1,2, \ldots, \max )$, eq. (1) (3) could be transformed into the following aggregation model.

Objective function:

$$
G=\min \sum_{k=1}^{B Z} \sum_{j=1}^{J Z} F_{k j}\left(W_{k j}\right)
$$

Water quantity constraint:

$$
\sum_{k=1}^{B Z} \sum_{j=1}^{J Z} W_{k j} \geq W_{e}
$$

Power constraint:

$$
N_{k j i}\left(\theta_{k j i}\right) \leq N_{k j 0}
$$

Taking $B Z$ pump stations as a suppositional station with $A Z$ pump units $(A Z=J Z \times B Z)$, eq. (15) (17) are also one-dimension dynamic programming model whose stage variable is $n(n=1,2, \ldots, A Z)$, the decision variable $W_{n}$ is the daily water quantity pumped by each unit, and the state variable $\lambda$ is the discrete value of water quantity pumped by all units. Applying dynamic programming method to solve the model above, the minimal daily electricity cost of entire parallel pumping group corresponding to the objective water quantity $W_{e}$ could be obtained, by which the optimal water quantity of each pump unit $W_{n}{ }^{*}(n=1,2, \ldots, A Z)$ could be obtained. The solving details of this model are similar to Chapter 2.2.2.

After getting a series of $W_{n}{ }^{*}$ values $(n=1,2, \ldots, A Z)$, by means of the results of solving the second-stage subsystem which is the optimal mathematical model of daily operation for single pump unit with adjustable-blade, we could get a series of optimal operation schemes of each pump unit which is the optimal blade angle $\theta_{i n}{ }^{*}(i=1,2, \ldots$, $S N ; n=1,2, \ldots, A Z)$ in each time period corresponding to each $W_{n}{ }^{*}(n=1,2, \ldots, A Z)$.

\subsection{Analysis of Optimal Operation for Multi-units with Adjustable-Blade in Huai'an Parallel Pumping Stations}

\subsubsection{Basic Informations of Huai'an Parallel Pumping Stations}

The basic information of No.1, No.2 and No.4 Huai' an Pumping Stations which are the second stage stations in the Eastern Route of the South-to-North Water Transfer Project is shown in Table 2. During the optimization process, one standby unit contained in No.4 Huai' an Pumping Station is not considered.

Table 2. Basic information of No.1, No.2 and No.4 Huai' an Pumping Stations

\begin{tabular}{cccccccc}
\hline $\begin{array}{c}\text { Pumping } \\
\text { station }\end{array}$ & $\begin{array}{c}\text { Type of } \\
\text { pump unit }\end{array}$ & $\begin{array}{c}\text { Unit } \\
\text { quantity }\end{array}$ & $\begin{array}{c}\text { Impeller } \\
\text { diameter } / \mathrm{mm}\end{array}$ & $\begin{array}{c}\text { Rated speed } \\
/ \mathrm{r} \cdot \mathrm{min}^{-1}\end{array}$ & $\begin{array}{c}\text { Match motor } \\
\text { power } / \mathrm{kW}\end{array}$ & $\begin{array}{c}\text { Rated blade } \\
\text { angle }{ }^{\circ}\end{array}$ & $\begin{array}{c}\text { Range of } \\
\text { adjustable- } \\
\text { blade }\end{array}$ \\
\hline No.1 & Axial-flow pump & 8 & 1640 & 250 & 1000 & 0 & $-4^{\circ} \sim+4^{\circ}$ \\
No.2 & Axial-flow pump & 2 & 4500 & 100 & 5000 & 0 & $-4^{\circ} \sim+4^{\circ}$ \\
No.4 & Axial-flow pump & 3 & 2900 & 150 & 2500 & 0 & $-4^{\circ} \sim+4^{\circ}$ \\
\hline
\end{tabular}


The upstream and downstream rivers of Huai'an parallel pumping stations have a big enough cubage, which makes the daily head has a small change scope. Therefor, with the assumption that the daily average head has a constant value, and within the feasible domain of parallel pumping stations, we disperse it into 6 average daily heads which are $3.13 \mathrm{~m}, 3.53 \mathrm{~m}, 3.93 \mathrm{~m}, 4.13 \mathrm{~m}, 4.53 \mathrm{~m}$ and $4.93 \mathrm{~m}$. In each of daily average head, full load, $80 \%$ load and $60 \%$ load of water quantity corresponding to operation with fixed blade angle and constant speed are considered as the optimal objective water quantity. Taking use of two-stage decomposition-dynamic programming aggregation method, we could get the electricity cost per $10^{4} \mathrm{~m}^{3}$ water quantity corresponding to minimal daily electricity cost of entire parallel pumping group under each daily average head and operation load.

\subsubsection{Optimization Results of Optimal Operation Model for Muilt-units with Adjustable-Blade in Huai'an Parallel Pumping Stations}

Making use of the method above, the optimal operation scheme of No.1, No.2 and No.4 Huai'an Pumping Station under each daily average head and operation load could be obtianed. Taking the daily average head of $4.13 \mathrm{~m}, 80 \%$ load for example, the optimal operation scheme is shown in Table 3 whose electricity cost per $10^{4} \mathrm{~m}^{3}$ water quantity is 79.84 yuan $/ 10^{4} \mathrm{~m}^{3}$. Fig. 1 shows the electricity cost per $10^{4} \mathrm{~m}^{3}$ water quantity of optimization under each operation load. And Fig. 2 shows the optimal water quantity allocation among all pmup units respectively under full load, $80 \%$ load and $60 \%$ load while the daily average head is $4.13 \mathrm{~m}$.

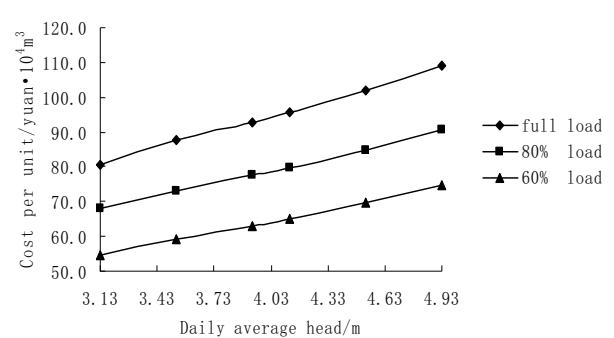

Fig. 1. Unit cost of water pumping under the optimal operation with adjustable -blade

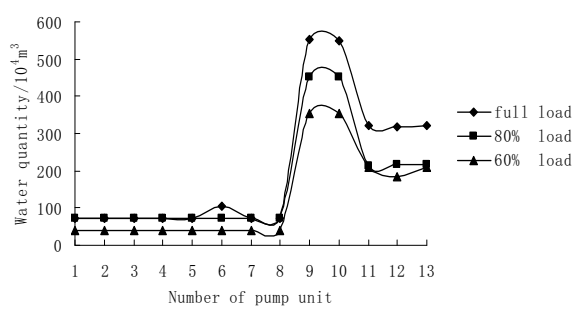

Fig. 2. Optimal water quantity allocation among units under different loads and daily average head of $4.13 \mathrm{~m}$

\subsubsection{Discussion of Optimization Results on Optimal Operation of Multi-units with Adjustable-Blade in Huai'an Parallel Pumping Stations}

Analyzing upon the figures and tables obtained from the optimization on multi-units with adjustable-blade in Huai' an parallel pumping stations by two-stage decomposition-dynamic programming aggregation method aiming to each daily average head and operation load, following results could be obtained.

(1) Average electricity cost per $10^{4} \mathrm{~m}^{3}$ water quantity of all daily average heads corresponding to full load, $80 \%$ load and $60 \%$ load operation are respectively 94.60 yuan $/ 10^{4} \mathrm{~m}^{3}, 78.98 \mathrm{yuan} / 10^{4} \mathrm{~m}^{3}$ and $64.37 \mathrm{yuan} / 10^{4} \mathrm{~m}^{3}$. 
Table 3. Optimal operation schemes of $80 \%$ load with adjustable-blade under daily average head of $4.13 \mathrm{~m}$ considering peak-valley electricity price

\begin{tabular}{|c|c|c|c|c|c|c|c|c|c|c|}
\hline $\begin{array}{l}\text { Pumping } \\
\text { station }\end{array}$ & $\begin{array}{l}\text { Unit } \\
\text { number }\end{array}$ & 1 & 2 & 3 & 4 & 5 & 6 & 7 & 8 & 9 \\
\hline \multirow{8}{*}{ No.1 } & Unit 1 & Stop & Stop & $0^{\circ}$ & $+1.5^{\circ}$ & $+1.5^{\circ}$ & Stop & Stop & $-2^{\circ}$ & $-2^{\circ}$ \\
\hline & Unit 2 & Stop & Stop & $0^{\circ}$ & $+1.5^{\circ}$ & $+1.5^{\circ}$ & Stop & Stop & $-2^{\circ}$ & $-2^{\circ}$ \\
\hline & Unit 3 & Stop & Stop & $0^{\circ}$ & $+1.5^{\circ}$ & $+1.5^{\circ}$ & Stop & Stop & $-2^{\circ}$ & $-2^{\circ}$ \\
\hline & Unit 4 & Stop & Stop & $0^{\circ}$ & $+1.5^{\circ}$ & $+1.5^{\circ}$ & Stop & Stop & $-2^{\circ}$ & $-2^{\circ}$ \\
\hline & Unit 5 & Stop & Stop & $0^{\circ}$ & $+1.5^{\circ}$ & $+1.5^{\circ}$ & Stop & Stop & $-2^{\circ}$ & $-2^{\circ}$ \\
\hline & Unit 6 & Stop & Stop & $0^{\circ}$ & $+1.5^{\circ}$ & $+1.5^{\circ}$ & Stop & Stop & $-2^{\circ}$ & $-2^{\circ}$ \\
\hline & Unit 7 & Stop & Stop & $0^{\circ}$ & $+1.5^{\circ}$ & $+1.5^{\circ}$ & Stop & Stop & $-2^{\circ}$ & $-2^{\circ}$ \\
\hline & Unit 8 & Stop & Stop & $0^{\circ}$ & $+1.5^{\circ}$ & $+1.5^{\circ}$ & Stop & Stop & $-2^{\circ}$ & $-2^{\circ}$ \\
\hline \multirow{2}{*}{ No.2 } & Unit 1 & $+2^{\circ}$ & $+2^{\circ}$ & $+4^{\circ}$ & $+4^{\circ}$ & $+4^{\circ}$ & Stop & Stop & $+4^{\circ}$ & $+4^{\circ}$ \\
\hline & Unit 2 & $+2^{\circ}$ & $+2^{\circ}$ & $+4^{\circ}$ & $+4^{\circ}$ & $+4^{\circ}$ & Stop & Stop & $+4^{\circ}$ & $+4^{\circ}$ \\
\hline \multirow{3}{*}{ No.4 } & Unit 1 & Stop & Stop & $+2^{\circ}$ & $+4^{\circ}$ & $+4^{\circ}$ & Stop & Stop & $+1^{\circ}$ & $+1^{\circ}$ \\
\hline & Unit 2 & Stop & Stop & $+1^{\circ}$ & $+4^{\circ}$ & $+4^{\circ}$ & Stop & Stop & $+2^{\circ}$ & $+4^{\circ}$ \\
\hline & Unit 3 & Stop & Stop & $+1^{\circ}$ & $+4^{\circ}$ & $+4^{\circ}$ & Stop & Stop & $+2^{\circ}$ & $+4^{\circ}$ \\
\hline
\end{tabular}

(2) Optimization results show that shut-down periods always appear in the period of high electricity price $(0.978$ yuan $/ \mathrm{kW} \cdot \mathrm{h})$ and while in operation period the high price corresponds to small blade angle of pump unit and vice versa. In the meantime, it is necessary to increase the shut-down periods instead of operating at the minus blade angle in order to save the electricity cost. That means there is a preferential consideration of controlling the number of operation units, after which the adjustable-blade measure would be taken.

(3) Fig. 2 shows that as a result of higher unit performance of No.2 Huai' an pumping station, the water quantity distributed to No.2 station is more that the others, which reflects efficiency priority principle.

(4) As the two-stage decomposition-dynamic programming aggregation method firstly takes optimal operation calculation for single pump unit with adjustable-blade by means of dynamic programming, after which the general coordination of water quantity by means of the aggregation model is taken, we could obtain optimal operation mode under the different blade angles of each pump unit in the same time period. Therefore, this method is suitable for solving the optimal daily operation problems of parallel pumping stations with different daily average heads, different time period divisions and different adjustable mode of pump unit in each pumping station.

\section{Conclusion}

Two-stage decomposition-dynamic programming aggregation method is first put forward to solve the optimal mathematical model of daily operation for multi-units with adjustable-blade in parallel pumping stations, by which the notable optimization results 
could be obtained. This method has a general guiding significance for the optimization problems of complex nonlinear mathematical models which are similar to eq. (1) (3), and could solve the optimal daily operation problems of parallel pumping stations with different daily average heads, different time period divisions and different adjustable mode of pump unit in each pumping station. Besides, a set of optimal operation schemes of parallel pumping stations under different daily average heads and operation loads have been established by means of calculatiing typical parallel pumping stations, which could offer references for the optimal operation of parallel pumping stations with small daily average head amplitude, and also make the study basis for the optimal operation for multi-stage pumping stations.

\section{References}

1. Liu, J., Zhang, Z., Zhang, M., et al.: Determination of economical operation program at draining pump station. J. Drainage and Irrigation Machinery 24(6), 20-23 (2006)

2. Ghassemi-Tari, F., Jahangiri, E.: Development of a hybrid dynamic programming approach for solving discrete nonlinear knapsack problems. J. Science Direct 188, 1023-1030 (2007)

3. Chen, S., Rui, J., Xu, Q., et al.: Daily Optimal Operation for Pumping Stations. J. Hydroelectric Energy 21(3), 82-83 (2003)

4. Zhu, H., Xia, F.: Application of decomposition-aggregation method of large-scale system on whole structural optimization of arch aqueduct. Journal of Hydraulic Engineering 10, 1-7 (1995)

5. Hassan, S., Ljubomir, G.: Decomposition-aggregation method applied to a multimachine power system. J. Large Scale Systems 10(2), 115-132 (1986)

6. Cheng, J., Zhang, L., Zhang, R., et al.: Study on optimal day-operation of single adjustable-blade pump unit. Journal of Hydraulic Engineering 41(4), 499-504 (2010)

7. Wang, D., Dong, Z., Ding, S.: Research on aggregation-decomposition-coordination model of feeding reservoir group. Journal of Hohai University (Natural Sciences) 34(6), 622-626 (2006)

8. Cooper, L., Cooper, M.W.: Introduction to Dynamic Programming. Pergamon Press, New York (1981) 